\title{
Opioid Measurement
}

National Cancer Institute

\section{Source}

National Cancer Institute. Opioid Measurement. NCI Thesaurus. Code C74697.

The determination of the amount of any opioid, including any of the naturally occurring and semi-synthetic opiates, fully synthetic opioids and their metabolites, present in a sample. 